\title{
Comunicación
}

\section{Calidad del Semen Refrigerado de Carneros Assaf y Blackbelly}

\author{
Quality of Refrigerated Assaf And Blackbelly Ram Semen
}

\author{
Jorge Isaac Castro Bedriñana ${ }^{1,2}$, Doris Maritza Chirinos Peinado', \\ Javier Angel Orellana Chirinos ${ }^{1}$
}

\section{Resumen}

Se comparó la calidad del semen refrigerado de carneros Assaf y Blackbelly utilizados en inseminación artificial y criados en la costa central del Perú. El semen fue colectado con vagina artificial y diluido en Tris-fructosa-yema de huevo. Se determinó la motilidad individual progresiva, la integridad de membrana mediante la prueba hipoosmótica (HOST) y la concentración espermática a través de la cámara de Neubauer a las dos horas de la refrigeración. Los valores porcentuales fueron transformados a ArcSeno $\sqrt{ } \%$. Se realizaron pruebas de «t $»$ Student para determinar diferencias de medias independientes entre las variables determinadas, según la raza. La motilidad individual progresiva fue de $82.36 \pm 6.66$ y $83.30 \pm 4.92 \%(p>0.05)$ y la motilidad masal fue de $4.28 \pm 0.45$ y $4.06 \pm 0.33(\mathrm{p}<0.05)$ para los carneros Assaf y Blackbelly, respectivamente. La integridad de la membrana espermática fue de $83.50 \pm 2.77 \%$ para Assaf y $82.56 \pm 3.78 \%$ para Blackbelly $(\mathrm{p}<0.05)$. La concentración espermática y los porcentajes de espermatozoides vivos y anormales fueron similares en ambas razas.

Palabras clave: calidad de semen; motilidad masal; inseminación artificial; semen refrigerado; concentración espermática

\section{Abstract}

Chilled semen quality of Assaf and Blackbelly rams used in artificial insemination and raised in the central coast of Peru were compared. Semen was collected with an artificial vagina and diluted with Tris-fructose-egg yolk. The individual progressive motility, the membrane integrity by the hypoosmotic text (HOST), and sperm

\footnotetext{
${ }^{1}$ Facultad de Zootecnia, Universidad Nacional del Centro del Perú, Huancayo, Perú

${ }^{2}$ E-mail: jorgecastrobe@yahoo.com
}

Recibido: 7 de noviembre de 2016

Aceptado para publicación: 30 de marzo de 2017 
concentration in the Neubauer chamber were determined after two hours of refrigeration. The percentage values were previously transformed into $\operatorname{ArcSen} \sqrt{ } \%$. The Student «t» test for independent means differences between breeds was performed. Progressive individual motility was $82.36 \pm 6.66$ and $83.30 \pm 4.92 \%(\mathrm{p}>0.05)$ and the mass motility was $4.28 \pm 0.45$ and $4.06 \pm 0.33(\mathrm{p}<0.05)$ for the Assaf and for Blackbelly rams, respectively. The integrity of the spermatic membrane was $83.50 \pm 2.77 \%$ for Assaf and $82.56 \pm 3.78 \%$ for Blackbelly $(\mathrm{p}<0.05)$. Sperm concentration and percentages of live and abnormal spermatozoa were similar in both breeds.

Key words: semen quality; mass motility; artificial insemination; chilled semen; sperm concentration

\section{INTRODUCCIÓN}

Entre las razas de ovinos introducidas al país, Assaf y Blackbelly tienen un buen desempeño en productividad y eficiencia (Atto, 2007); no obstante, para establecer programas de poblamiento que consideren el uso de la inseminación artificial, la calidad del semen debe estar adecuadamente evaluada (David et al., 2015). Las características macroscópicas y microscópicas del semen de ovinos han sido estudiadas (Evans y Maxwell, 1987; Gibbons et al., 1993; Ramírez, 2002; Bezerra y Soto-Blanco, 2010) y los protocolos propuestos se vienen empleando en estudios de evaluación seminal. Asimismo, las asociaciones entre la raza de los carneros y la calidad del semen (Abdel-Rahman et al., 2000; Rege et al., 2000; Gundogan y Demirci, 2003; Salhab et al., 2003; David et al., 2007; Domínguez, et al., 2008), así como los efectos de diversos dilutores sobre la motilidad individual y progresiva (Gibbons et al., 1993; Salamon y Maxwell, 2000; Vishwanath y Shannon, 2000; Cabrera et al., 2010) y sobre la integralidad funcional de la membrana espermática (Stornelli, 2005; Cabrera et al., 2010) vienen evaluándose en diversos estudios.

El test de endosmosis (Hypoosmotic Swelling Test - Host) permite la comprobación del estado funcional de la membrana plasmática, de allí que podría utilizarse como valor predictivo de fertilidad (Pérez-Llano et al., 2003). Los valores de Host se correla- cionan con otros parámetros de calidad seminal, como la motilidad, viabilidad o la morfología, permitiendo predecir la capacidad fecundante del semen (Rota et al., 2000).

El semen de ovinos Assaf presentan concentraciones entre 1780 y 3990 × $10^{6}$ espermatozoides por mililitro (Epstein, 1985), en tanto que para el semen de carneros Blackbelly, Quispe (1998) reporta 3730 x $10^{6}$ espermatozoides $/ \mathrm{ml} \mathrm{y} 1.46 \mathrm{ml}$ de volumen de eyaculado con $83 \%$ de motilidad individual, mientras que Guillén (2001) reporta 84.9\% de motilidad individual, $3140 \times 10^{6}$ de espermatozoides $/ \mathrm{ml}, 0.97 \mathrm{ml}$ de volumen de eyaculado y 6.6-6.8 de $\mathrm{pH}$.

Considerando la importancia que tienen las razas Assaf y Blackbelly en la producción de ovinos en la costa y la selva peruana, el objetivo de la presente investigación fue comparar las características macroscópicas y microscópicas del semen, la integridad de las membranas (Host) y la motilidad individual progresiva (MIP) de los espermatozoides en carneros de ambas razas, luego de un periodo de refrigeración de dos horas.

\section{Materiales y Métodos}

La investigación se llevó a cabo en el Banco Nacional de Semen de la Universidad Nacional Agraria La Molina (UNALM), situada en Lima, costa subtropical del Perú, con una temperatura promedio anual de $18^{\circ} \mathrm{C}$ 
y una humedad relativa anual promedio de $75 \%$.

Se utilizaron tres carneros Assaf y tres Blackbelly, de 3.5 años de edad en promedio, pertenecientes a la granja Rigoranch, UNALM, los cuales tuvieron el mismo manejo zootécnico y fueron alimentados con king grass y maíz chala picada, más un suplemento concentrado, ofrecidos diariamente en cantidades similares a cada carnero. Los animales estuvieron alojados en corrales de 2.8 x $3.0 \times 1.2 \mathrm{~m}$, provistos de sombra, comedero y bebedero. Las instalaciones tuvieron una adecuada iluminación y ventilación. En cada corral se colocaron dos carneros.

Se hicieron dos colecciones de semen por semana, y un total de 12 colecciones por carnero para los fines del experimento. Hubo una fase de dos semanas de entrenamiento a la colección de semen con vagina artificial. Los eyaculados fueron colocados en baño maría a $34^{\circ} \mathrm{C}$. Se utilizó Tris-fructosa-yema de huevo como dilutor, cuya composición consideró Tris $(3.63 \mathrm{~g})$, fructosa $(0.5 \mathrm{~g})$, ácido cítrico (1.95 g), yema de huevo (15 ml), penicilina (100 000 UI), estreptomicina (100 $\mathrm{mg})$, agua destilada $(80 \mathrm{ml})$ y glicerol $(5 \mathrm{ml})$, siguiendo el protocolo de Evans y Maxwell (1987).

La valoración cuantitativa del movimiento de los espermatozoides se determinó inmediatamente después de la colección de semen, siguiendo el protocolo de Evans y Maxwell (1987), empleando la escala de 0 a 5. La motilidad individual progresiva (0$100 \%$ ) fue determinada con base a los protocolos de Gibbons et al. (1993), empleando la cámara de Neubauer. La integridad funcional de membrana espermática se evaluó mediante la prueba hipoosmótica (HOST), siguiendo el protocolo de Correa y Zavos (1994). La morfología de los espermatozoides se determinó mediante el método de frotis de semen teñido, utilizando eosina y nigrosina al 5 y $10 \%$, respectivamente, observando no menos de 300 espermatozoides al microscopio (400X). El porcentaje de espermatozoides vivos se determinó considerando el número porcentual de espermatozoides anormales en relación al número de espermatozoides contados.

La motilidad masal, concentración espermática, porcentaje de espermatozoides vivos y el porcentaje de espermatozoides anormales, HOST refrigerado y MIP-tris-refrigerado del semen de carneros Assaf y Blackbelly fueron comparadas mediante pruebas de «t $\rangle$ para diferencia de medias independientes, empleando el software estadístico SAS (Statistical Analysis System, v. 8.0). Los valores porcentuales fueron previamente transformados angularmente, Arco Seno $\sqrt{ } \%$ (Snedecor y Cochrane, 1989).

\section{Resultados y Discusión}

El volumen de eyaculado de carneros Assaf fue mayor que el de carneros Blackbelly ( $p<0.05$, Cuadro 1$)$. Los volúmenes encontrados se encuentran en el rango normal de eyaculados en ovinos (Hafez (1996). Asimismo, Quispe (1998) reporta volúmenes de $1.46 \mathrm{ml}$, mientras que Guillén (2001) y Ramírez (2002) reportan eyaculado de $0.97 \mathrm{ml}$ entre 0.83 y $0.97 \mathrm{ml}$ en ovinos Blackbelly criados en Lima, Perú.

Todos los eyaculados fueron de color blanco cremoso, con un pH de 7.0, valor adecuado para el semen de ovinos (Evans y Maxwell, 1987). Por su parte, Guillén (2002) en carneros Blackbelly del Programa de Crianzas Familiares del Instituto Nacional de Innovación Agraria (INIA), Lima, reporta valores de $\mathrm{pH}$ ligeramente inferiores al registrado en el presente estudio (6.6-6.8).

En el Cuadro 1 se muestran los resultados correspondientes a las características microscópicas del semen de los carneros Assaf y Blackbelly. La motilidad masal fue alta en las dos razas, pero estadísticamente mayor en los carneros Assaf $(p<0.05)$. Asimismo, los valores fueron similares a los re- 
Cuadro 1. Volumen del eyaculado y características microscópicas del semen de carneros Assaf y Blackbelly de 3.5 años de edad ( 3 carneros por raza y 12 eyaculados por carnero)

\begin{tabular}{llccccc}
\hline Variable & Raza & Promedio & d.e. & $\begin{array}{c}\text { CV } \\
(\%)\end{array}$ & Mín. & Máx. \\
\hline \multirow{2}{*}{ Volumen (ml) } & Assaf & $1.92^{\mathrm{a}}$ & 0.69 & & 1.4 & 3.2 \\
& Blackbelly & $1.50^{\mathrm{b}}$ & 0.56 & & 0.6 & 1.8 \\
Motilidad masal (0-5) & Assaf & $4.28^{\mathrm{a}}$ & 0.45 & 10.51 & 4.0 & 5.0 \\
& Blackbelly & $4.06^{\mathrm{b}}$ & 0.33 & 8.13 & 4.0 & 5.0 \\
Concentración espermática & Assaf & $2695^{\mathrm{a}}$ & 764 & 28.34 & 1260 & 4600 \\
(N. espermatozoides x 10 $)$ & Blackbelly & $2459^{\mathrm{a}}$ & 904 & 36.75 & 1320 & 4760 \\
Espermatozoides vivos (\%) & Assaf & $81.89^{\mathrm{a}}$ & 5.97 & 7.29 & 67.0 & 90.0 \\
& Blackbelly & $80.19^{\mathrm{a}}$ & 5.87 & 7.32 & 70.0 & 95.4 \\
Espermatozoides anormales & Assaf & $7.11^{\mathrm{a}}$ & 2.05 & 28.83 & 4.0 & 12.0 \\
& Blackbelly & $6.94^{\mathrm{a}}$ & 1.57 & 22.62 & 5.0 & 11.0 \\
\hline
\end{tabular}

$a, b$ Valores promedio por variable por raza con letras diferentes son estadísticamente diferentes $(\mathrm{p}<0.05)$

Cuadro 2. Características microscópicas del semen refrigerado con diluyente tris-fructuosayema de huevo en carneros Assaf y Blackbell de 3.5 años de edad ( 3 carneros por raza y 12 eyaculados por carnero)

\begin{tabular}{llccccc}
\hline Variable & Raza & Promedio & d.e. & CV (\%) & Mín. & Máx. \\
\hline Host $^{1}$ & Assaf & $83.50^{\mathrm{a}}$ & 2.77 & 3.32 & 78 & 89 \\
Host & Blackbelly & $82.56^{\mathrm{b}}$ & 3.78 & 4.58 & 70 & 89 \\
MIP-tris $^{2}$ & Assaf & $82.36^{\mathrm{a}}$ & 6.66 & 8.09 & 55 & 94 \\
MIP-tris & Blackbelly & $83.30^{\mathrm{a}}$ & 4.92 & 5.91 & 71 & 91 \\
\hline
\end{tabular}

a,b Valores promedio por variable y raza con letras diferentes varían estadísticamente $(p<0.05)$

${ }^{1}$ Prueba hipoosmótica

${ }^{2}$ Motilidad individual progresiva

portados por Cabrera et al. (2010), quiénes también encontraron diferencias significativas a favor de los carneros Assaf.

La mayor motilidad masal en carneros Assaf $(\mathrm{p}<0.05)$ podría atribuirse a factores genéticos (Gundogan et al., 2004) y ambientales (Abdel-Rahman et al., 2000; Rege et al., 2000; Gundogan et al., 2004), antecedentes nutricionales (Toe et al., 1994) y varia- ciones estacionales (Ibrahim, 1997; Rege et al., 2000; Gundogan y Demirci, 2003; Salhab et al., 2003; Domínguez et al., 2008).

La concentración espermática fue similar entre las dos razas, estando los promedios dentro del rango reportado por Epstein (1985) para carneros Assaf, mientras que promedios inferiores a los reportados por Quispe (1998) y Guillén (2001) para carneros 
Blackbelly. Por otro lado, Cabrera et al. (2010) refiere valores similares a los del presente estudio, pero con diferencias significativas entre estas razas.

El porcentaje de espermatozoides vivos $\mathrm{y}$ anormales en ambas razas fueron similares $\mathrm{y}$ con valores que permiten afirmar que el semen colectado fue de buena calidad, tanto por sus características macroscópicas y microscópicas; los mismos que se encuentran dentro del rango reportado por Hafez (1996). El alto porcentaje de espermatozoides vivos guarda relación con el movimiento progresivo.

En el Cuadro 2 se presentan las características del semen diluido y refrigerado. El valor de Host fue significativamente más alto en los carneros Assaf $(\mathrm{p}<0.05)$, mientras que la motilidad progresiva fue similar para ambas razas.

Los valores de la prueba de Host superaron el $82 \%$, valor que determina una alta capacidad fecundante de los espermatozoides (Hafez, 1996; Rota et al., 2000; Pérez-Llano et al., 2003). Los valores de Host fueron similares a los reportados por Cabrera et al. (2010) para carneros Assaf $(83.3 \pm 5.9)$ y Blackbelly $(81.4 \pm 3.2)$ en semen fresco.

La motilidad individual progresiva (MIP) del semen refrigerado fue similar a otros reportes en semen de carneros Blackbelly (Quispe, 1998; Guillén, 2001; Ramírez, 2002), aunque ligeramente inferiores a los valores reportados por Cabrera et al. (2010) de 84.5 \pm 3.0 para Assaf y $83.7 \pm 3.3$ para Blackbelly.

\section{Conclusiones}

- Los carneros de la raza Assaf registraron un mayor volumen seminal, motilidad masal e integridad de membrana espermática que los carneros Blackbelly $(p<0.05)$, no habiendo diferencias en concentración espermática, porcentaje de espermatozoides vivos y motilidad individual progresiva.
- El factor raza del carnero no fue influyente ni determinante en relación a las características seminales durante la refrigeración.

\section{Agradecimiento}

Al Ing. MSc Próspero Cabrera Villanueva, Jefe del Banco Nacional de Semen durante le ejecución del trabajo, por haber brindado las facilidades necesarias para el desarrollo del estudio.

\section{Literatura Citada}

1. Abdel-Rahman HA, El-Belely MS, AlQarawi AA, El-Mougy SA. 2000. The relationship between semen quality and mineral composition of semen in various ram breed. Small Rumin Res 38: 45-49. doi: 10.1016/S0921-4488(00)00137-1

2. Atto MJ. 2007. Importancia de los ovinos tropicales introducidos al país: características productivas y reproductivas. Arch Latinoam Prod Anim 15 (Supl 1): 310-315.

3. Bezerra FC, Soto-Blanco B. 2010. Sperm quality of sheep fed cottonseed cake. Acta Sci Vet 38: 415-418.

4. Cabrera VP, Orellana J, Pantoja AC. 2010. Efecto de dos dilutores sobre la motilidad e integridad de la membrana espermática en semen congelado de ovinos. Rev Inv Vet Perú 21: 154-160. doi: 10.15381/rivep.v21i2.130

5. Correa J, Zavos P. 1994. The hypoosmotic swelling test: its employment as an assay to evaluate the functional integrity of the frozen-thawed bovine sperm membrane. Theriogenology 42: 351-360.

6. David I, Bodin L, Lagriffoul G, Manfredi E, Robert-Granie C. 2007. Character process model for semen volume in AI rams: evaluation of correlation structures for long and shortterm environmental effects. Genet Sel Evol 39: 55-71. doi: 10.1051/gse:2006033 
7. David I, Kohnke P, Lagriffoul G, Praud O, Plouarboué P, Degond P, Druart $X$. 2015. Mass sperm motility is associated with fertility in sheep. Anim Reprod Sci 161: 75-81. doi: 10.1016/ j.anireprosci.2015.08.006

8. Domínguez MP, Falcinelli A, Hozbor F, Sánchez E, Cesari A, Alberio RH. 2008. Seasonal variations in the composition of ram seminal plasma and its effect on frozen-thawed ram sperm. Theriogenology 69: 564-573. doi: 10.1016/j.theriogenology.2007.11.010

9. Epstein H. 1985. The Awassi sheep with special reference to the improved dairy type. FAO Animal Production and Health Paper $N^{\circ} 57.84$ p. [Internet]. Disponible en http://www.fao.org/ docrep/011/aj003e/AJ003E00.htm

10. Evans G, Maxwell WM. 1987. Salamon's artificial insemination of sheep and goats. UK: Butterworth. 122 p.

11. Gibbons A, Cueto M, Wolff M, Arrigo J, García J. 1993. Obtención, procesamiento y conservación del semen de ovino. Comunicación Técnica de Producción Animal del INTA. Bariloche $\mathrm{N}^{\circ} 443$.

12. Guillén H. 2001. Evaluación de las características seminales en carneros Blackbelly. Tesis de Ing. Zootecnista. Lima: Universidad Nacional Agraria La Molina. 68 p.

13. Gundogan M, Demirci F. 2003. Monthly changes in some reproductive parameters and in testosterone and thyroxin values of rams throughout one year under continental climate conditions. Dtsch Tieraztl Wochenschr 110: 450-453.

14. Gundogan M, Yeni D, Ucar M, Ozenc E. 2004. Relationship between some reproductive parameters and biochemical properties of blood serum in ram. Arch Androl 50: 387-390. doi: 10.1080/01485010490474634

15. Hafez ESE. 1996. Reproducción e inseminación artificial en animales. $6^{\mathrm{a}}$ ed. México: Interamericana. 542 p.
16. Ibrahim SA. 1997. Seasonal variations in semen quality of local and crossbred raised in the United Arab Emirates. Anim Reprod Sci 49: 161-167. doi: 10.1016/ S0378-4320(97)00063-8

17. Pérez-Llano B, Yenes-García P, García-Casado P. 2003. Four subpopulations of boar spermatozoa defined according to their response to the short hypoosmotic swelling test and acrosome status during incubation at $37^{\circ} \mathrm{C}$. Theriogenology 60: 1401-1407.

18. Quispe PE. 1998. Natalidad y prolificidad en ovejas Assaf y Assaf x Blackbelly sincronizadas e inseminadas con semen fresco y congelado. Tesis de Maestría. Lima: Universidad Nacional Agraria La Molina. $98 \mathrm{p}$

19. Ramírez CA. 2002. Efecto de tres dilutores y tres tiempos de refrigeración en la motilidad individual del semen refrigerado de carneros Black Belly. Tesis de Ing. Zootecnista. Lima: Universidad Nacional Agraria La Molina. 93 p.

20. Rege JEO, Toel F, Mukasa-Mugerwa E, Tembely S, Amindo D, Baker RL, Lahlou-Kassi A. 2000. Reproductive characteristics of Ethiopian highland sheep. II. Genetic parameters of semen characteristics and their relationships with testicular measurements in ram lambs. Small Rumin Res 37: 173-187. doi: 10.1016/S0921-4488(00)00140-1

21. Rota A, Penso N, Vicenti L, Mantovani R. 2000. Hyposmotic swelling (HOS) as a screening assay for testing in vitro fertility of bovine espermatozooa. Theriogenology 53: 1415-1420. doi: 10.1016/S0093-691X(00)00284-3

22. Salamon S, Maxwell WM. 2000. Storage of ram semen. Anim Reprod Sci 62: 77-111. doi: 10.1016/S0378-4320(00) 00155-X

23. Salhab SA, Zarkawi M, Wardeh MF, Al-Masri MR, Kassem R. 2003. Characterization and evaluation of semen in growing Awassi ram lambs. Trop Anim Health Prod 35: 455-463. doi: 10.1023/ A:1025823730733 
24. Snedecor GW, Cochrane WG 1989. Statistical methods. $8^{\text {th }}$ ed. Iowa, USA: Iowa State University Press. 503 p.

25. Stornelli MC, Tittarelli CM, Savignone CA, Stornelli MA. 2005. Efecto de los procesos de criopreservación sobre la fertilidad seminal. Analecta Vet 25(2): 28-35.

26. Toe F, Lahlou-Kassi A, MukasaMugerwa E. 1994. Semen characte- ristics of Ile-de-France rams of different age and physical condition. Theriogenology 42:321-326. doi: 10.1016/0093691X(94)90276-3

27. Vishwanath R, Shannon P. 2000. Storage of bovine semen in liquid and frozen state. Anim Reprod Sci 62: 23-53. doi: 10.1016/ S0378-4320(00)00153-6 